\title{
Microbiota patterns and risk of cardiometabolic disease - opportunities for intervention?
}

\author{
Peter M. Nilsson \\ Lund University, Sweden
}

\begin{abstract}
In every human being, there is a substantial proportion (1-2 kg) of total body weight constituted by the gut microbiota content in the gastro-intestinal system. Recent research findings, based on mapping of the microbiome, have stated that in healthy subjects, the gut microbiota richness and diversity is higher as compared to obese subjects or patients suffering from cardiometabolic disease, or even long-standing hypertension when a different and less rich pattern is seen (dysbiosis). Intervention with certain food constituents, e.g. Mediterranean diet or dairy products such as lactobacillae-containing yoghurt, holds promise that this could be of benefit for improved organ function, improved metabolism and lowering disease risk. Randomized, controlled intervention studies are needed to test the hypothesis that a reduction in dysbiosis induced by external dietary interventions could translate into health benefits. Key words: microbiota, cardiometabolic disease

Arterial Hypertens. 2016, vol. 20, no. 1, pages: 1-4 DOI: 10.5603/AH.2016.0001
\end{abstract}

In cardiovascular epidemiology, the major traditional risk factors have been known for decades (hypertension, hyperlipidemia, smoking, diabetes), but in later years the interest grew for new risk markers such as insulin resistance, chronic inflammation, albuminuria and the metabolic syndrome, just to name a few. These risk factors act on increasing pathophysiological processes leading to target organ damage (TOD) that is measurable as the consequence of the risk burden during many years, such as left ventricular hypertrophy (LVH), arterial stiffness (increased pulse wave velocity, PWV), microalbuminuria and white matter lesions in the central nervous system. Modern guidelines on cardiovascular prevention have tried to discuss the practical implications of these risk factors and corresponding complications $[1,2]$.

A positive family history of cardiovascular disease is a component of all these guidelines and a widely studied factor for increased risk, especially if there is early onset of disease manifestations in first-degree relatives or other close family members [3]. The epidemiological background is that a number of chronic disease conditions tend to cluster in families with an increased risk in first-degree relatives, but also an increased risk in second-degree relatives [4]. This fact is most often referred to as the heritability (heredity) of these diseases and explained by the influence of genetic factors transferred across generations, or shared environment, even if the more specific details or mechanisms leading to disease are not known. In clinical medicine, a positive family history is often asked about during consultations, but the information provided is sometimes not exact, due to unawareness, poor recollection of data or other factors which bias the answers. Therefore, new methods have to be explored in screening studies and register linkage studies to define and measure consequences of a positive family history of disease. Furthermore, recent studies have indicated that different genetic scores of well-known genetic risk markers of disease conditions such as obesity, type 2 diabetes or myocardial infarction are only able to explain a minor proportion of this described heri-

Address for correspondence: Peter M. Nilsson, Professor, MD, $\mathrm{PhD}$

Dept. Clinical Sciences, Lund University, IM Nilssons gata 32, 2nd floor, Skåne University Hospital, Malmö, Sweden e-mail: Peter.Nilsson@med.lu.se 
tability [5]. Thus, there is still a lack of knowledge to explain the so-called "missing heritability" of these disorders. One model for understanding this is that gene-environmental interactions and epigenetics will add information to explain the hereditability, besides influences of shared environment in a broader sense. This is still not a well-researched area, which is why there is a need of more and better quality information from both populations and families on genetic profiling (genotyping) as well as on bodily function (phenotyping) and data on lifestyle and environmental exposures. Of special relevance is to elucidate on genetic and non-genetic mechanisms behind early cardiovascular and metabolic ageing [6], as a model for early disease onset within risk families. These associations are now being investigated in the ongoing Malmö Offspring Study (www.med.lu.se/mos) in southern Sweden, including genetic mapping as well as advanced phenotyping across three generations. In addition, a 4-day dietary registration is being carried out, by use of a web-form as well as by other methods.

\section{The gut microbiota pattern in health and disease}

Of particular interest is to determine the role of the microbiome, measured as microbiota pattern (the gut bacteria composition and diversity, but also from the oral cavity), and its association with dietary intake together with the genetic profile of the host, in relation to alterations in metabolism, obesity, hypertension, vascular function and immunological function [7-10]. This will be linked to other research areas in microbiology, nutritional sciences, technology, and innovation for prevention. For example, functional food products can be developed and tailored to match the profile and needs of the individual based on a more detailed personal profiling, for example the nutrition-gene-microbiota mapping (stratified medicine).

A remarkable finding in a family perspective is that the microbiome of individuals is influenced in early life, in the first place by the microbiota of the mother from exposure to the offspring during vaginal delivery and during the neonatal period [11]. Later on, this is influenced by more or less shared microbiota patterns in the family household during childhood and adolescence due to cohabitation. It is hypothesized whether microbiota profile as well as dietary intake patterns may cluster within families. This pattern is likely to change temporarily, following travelling abroad or treatment periods with antibio- tics, but may return after some time to the previous (programmed) pattern.

\section{Biomarkers of diet-microbiota patterns may predict future cardiovascular outcomes}

Recently, it has been discovered that there is also a specific serum biomarker, the pro-atherosclerotic metabolite, trimethylamine- $\mathrm{N}$-oxide (TMAO) that is able to reflect the gut microbiome and predict cardiovascular events in healthy subjects [12]. TMAO can also predict prognosis in patients with congestive heart failure, thus influencing clinical prognosis [13]. Interventions to change the microbiota have provided promising results [14] and been described as a potential new treatment target for cardio-metabolic disease. Such interventions should be based on the wider use of designed and tested functional food products as part of a healthy lifestyle in general. This provides an ultimate test on the causality linking microbiota patterns and disease and whether this association is reversible or not following interventions.

\section{Microbiota patterns in subjects with hypertension}

The microbiota pattern in association with hypertension has been documented in both hypertensive animal models [15] as well as in human hypertensives $[16,17]$. In one of the best studies so far, bacterial DNA from fecal samples of two rat models of hypertension and a small cohort of hypertensive patients, was used for bacterial genomic analysis [16]. The authors observed a significant decrease in microbial richness, diversity, and evenness in the spontaneously hypertensive rat, in addition to an increased Firmicutes/Bacteroidetes ratio. In addition, the microbiota of a small cohort of human hypertensive patients was found to follow a similar dysbiotic pattern, as it was less rich and diverse than that of control subjects, and with a microbiota pattern not overlapping that of the normotensive controls. Similar changes in gut microbiota were observed in the chronic angiotensin II infusion rat model, most notably decreased microbial richness and an increased Firmicutes/Bacteroidetes ratio. In this lab model, the efficacy of an oral antibiotic drug (minocycline) in restoring gut microbiota was also tested. In addition to attenuating high blood pressure, minocycline treatment was able to rebalance 


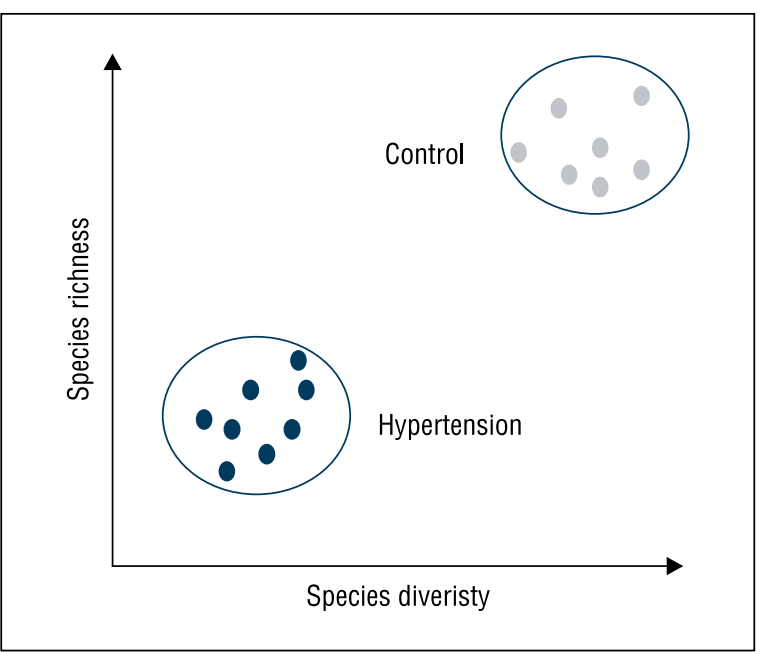

Figure 1. Schematic representation of microbiota patterns in patiens with hypertension compared with control. Samples derived from patiens with hypertension formed separate cluster and show significant decrese in species richness and diversity compared to healthy control samples (Figure was prepared based on Yang et al. Hypertension 2015; 65: 1331-1340. DOI: 10.1161/HYPERTENSIONAHA.115.05315)

the dysbiotic hypertension gut microbiota by reducing the Firmicutes/Bacteroidetes ratio. Thus, these observations clearly demonstrated that high blood pressure is associated with gut microbiota dysbiosis, both in animal and human hypertension [16]. However, larger studies are needed, as well as inclusion of hypertensive patients of different disease duration and prevalence rates of TOD.

\section{Could gut microbiota patterns be favorably intervened on?}

Different dietary interventions influencing gut microbiota have been tried in hypertension. One meta-analysis could show that increased intake of dairy products, including lactobacillae-containing yoghurt, is associated with a reduced tendency to develop hypertension during the follow-up period [18].

In another study, it was shown that blueberries fermented with the tannase-producing bacteria $L$. plantarum DSM 15313 have anti-hypertensive properties and may reduce the risk for cardiovascular diseases [19].

Finally, the anti-inflammatory properties of the Mediterranean diet could be another mode of action to explain the health benefits and cardiovascular prevention possible to achieve with implementing this diet [20], a link that has been proposed to change gut microbiota patterns for the better [21]. In this perspective, there seems to exist a considerable potential for dietary modifications influencing gut microbiota composition and facilitating health [22].

\section{Summary}

In summary, the family clustering of cardiometabolic disorders is a well-known clinical observation, but still not fully understood. Genetic factors, even put together in a genetic risk score, can explain just a minor proportion of the increased family risk. New discoveries linking gut microbiota to obesity, type 2 diabetes and cardiometabolic risk conditions could explain some of this increased clustering. Gut microbiota could potentially be influenced by functional food products, probiotics and healthy lifestyle, providing a link to cardiovascular and metabolic prevention. The Mediterranean diet could be of special importance for health benefits and its associations to the gut microbiota patterns should be explored. In a similar way the health aspects, as mediated by gut microbiota influences, of pickled vegetables ("kapusta") rich in lactobacillae as well as dairy products such as natural yoghurt should be further explored.

Funding: The Malmö Offspring Study (MOS) is funded by the Research Council of Sweden and the Heart- and Lung foundation.

Acknowledgements: I would like to thank Professor Marju Orho-Melander for fruitful discussion on the importance of microbiota patterns in health and disease.

\section{References}

1. Perk J., De Backer G., Gohlke H. et al.; European Association for Cardiovascular Prevention \& Rehabilitation (EACPR); ESC Committee for Practice Guidelines (CPG). European Guidelines on cardiovascular disease prevention in clinical practice (version 2012). The Fifth Joint Task Force of the European Society of Cardiology and Other Societies on Cardiovascular Disease Prevention in Clinical Practice (constituted by representatives of nine societies and by invited experts). Eur. Heart J. 2012; 33: 1635-1701

2. Mancia G., Fagard R., Narkiewicz K. et al:; Task Force Members. 2013 ESH/ESC Guidelines for the management of arterial hypertension: the Task Force for the management of arterial hypertension of the European Society of Hypertension (ESH) and of the European Society of Cardiology (ESC). J. Hypertens. 2013; 31: 1281-1357.

3. Ranthe M.F., Carstensen L., Oyen N. et al. Family history of premature death and risk of early onset cardiovascular disease. J. Am. Coll. Cardiol. 2012; 60: 814-821.

4. Zöller B., Li X., Sundquist J., Sundquist K. Multiplex sibling history of coronary heart disease is a strong risk factor for coronary heart disease. Eur. Heart J. 2012; 33: 2849-2855.

5. InterAct Consortium; Scott R.A., Langenberg C., Sharp S.J. et al. The link between family history and risk of type 2 diabetes is not explained by anthropometric, lifestyle or genetic risk factors: the EPIC-InterAct study. Diabetologia 2013; 56: 60-69.

6. Nilsson P.M., Boutouyrie P., Cunha P. et al. Early vascular ageing in translation: from laboratory investigations to clinical applications in cardiovascular prevention. J. Hypertens. 2013; 31: 1517-1526 .

7. Tremaroli V., Bäckhed F. Functional interactions between the gut microbiota and host metabolism. Nature 2012; 489: 242-249.

8. Cox A.J., West N.P., Cripps A.W. Obesity, inflammation, and the gut microbiota. Lancet Diabetes Endocrinol. 2015; 3: 207-215. 
9. Jose P.A., Raj D. Gut microbiota in hypertension. Curr. Opin. Nephrol. Hypertens. 2015; 24: 403-409.

10. Kotsis V., Nilsson P., Grassi G. et al.; WG on Obesity, Diabetes, the High Risk Patient, European Society of Hypertension. New developments in the pathogenesis of obesity-induced hypertension. J. Hypertens. 2015; 33: 1499-1508.

11. El Aidy S., Hooiveld G., Tremaroli V., Bäckhed F., Kleerebezem M. The gut microbiota and mucosal homeostasis: colonized at birth or at adulthood, does it matter? Gut Microbes. 2013; 4: 118-124.

12. Tang W.H., Wang Z., Levison B.S. et al. Intestinal microbial metabolism of phosphatidylcholine and cardiovascular risk. N. Engl. J. Med. 2013; 368: 1575-1584.

13. Tang W.H., Wang Z., Fan Y. et al. Prognostic value of elevated levels of intestinal microbe-generated metabolite trimethylamine- $\mathrm{N}$-oxide in patients with heart failure: refining the gut hypothesis. J. Am. Coll. Cardiol. 2014; 64: 1908-1914.

14. Ettinger G., MacDonald K., Reid G., Burton J.P. The influence of the human microbiome and probiotics on cardiovascular health. Gut Microbes. 2014; 5: 719-728.

15. Mell B., Jala V.R., Mathew A.V. et al. Evidence for a link between gut microbiota and hypertension in the Dahl rat. Physiol. Genomics 2015; 47: 187-197.
16. Yang T., Santisteban M.M., Rodriguez V. et al. Gut dysbiosis is linked to hypertension. Hypertension. 2015; 65: 1331-1340.

17. Kim S., Rodriguez V., Santisteban M. et al. 6B.07: Hypertensive patients exhibit gut microbial dysbiosis and an increase in TH17 cells (Abstract). J. Hypertens. 2015; 33 (suppl. 1): e77-8.

18. Soedamah-Muthu S.S., Verberne L.D., Ding E.L., Engberink M.F., Geleijnse J.M. Dairy consumption and incidence of hypertension: a dose-response meta-analysis of prospective cohort studies. Hypertension 2012; 60: 1131-1137.

19. Ahrén I.L., Xu J., Önning G., Olsson C., Ahrné S., Molin G. Antihypertensive activity of blueberries fermented by Lactobacillus plantarum DSM 15313 and effects on the gut microbiota in healthy rats. Clin Nutr. 2015; 34: 719-726.

20. Estruch R., Ros E., Salas-Salvadó J. et al.; PREDIMED Study Investigators. Primary prevention of cardiovascular disease with a Mediterranean diet. N. Engl. J. Med. 2013; 368: 1279-1290.

21. Del Chierico F., Vernocchi P., Dallapiccola B., Putignani L. Mediterranean diet and health: food effects on gut microbiota and disease control. Int. J. Mol. Sci. 2014; 15: 11678-11699.

22. Cotillard A., Kennedy S.P., Kong L.C. et al; ANR MicroObes consortium; Doré J., Zucker J.D., Clément K., Ehrlich S.D. Dietary intervention impact on gut microbial gene richness. Nature 2013; 500: 585-588. 